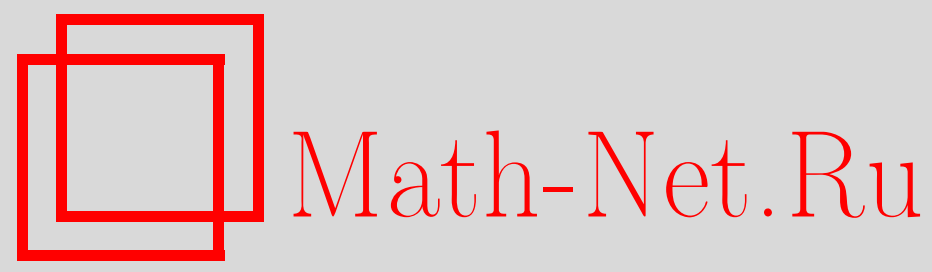

Д. Е. Ефимов, Оценка нормы разности между частичными произведениями некоторых последовательностей линейных операторов, Дискрет. матем., 2012, том 24, выпуск 3, 6672

DOI: https://doi.org/10.4213/dm1198

Использование Общероссийского математического портала Math-Net.Ru подразумевает, что вы прочитали и согласны с пользовательским соглашением http://www . mathnet.ru/rus/agreement

Параметры загрузки:

IP : 52.6 .47 .48

26 апреля 2023 г., 13:48:40 


\title{
Оценка нормы разности между \\ частичными произведениями некоторых последовательностей линейных операторов
}

\author{
() 2012 г. Д. Е. Ефимов
}

\begin{abstract}
Аналогично частичной сумме ряда, вводится понятие частичного произведения последовательности линейных операторов. Доказываются верхние оценки норм разностей между частичными произведениями некоторых последовательностей ограниченных линейных операторов в банаховом пространстве и верхние оценки сумм степеней сингулярных чисел операторов, получаемых как разность между частичными произведениями некоторых последовательностей ограниченных линейных операторов в сепарабельном гильбертовом пространстве. Результаты используются для оценки точности решений интегральных уравнений.
\end{abstract}

\section{1. Введение}

Векторное пространство $X$ с отмеченной нормой называется нормированным пространством. Нормированное пространство, полное относительно указанной метрики, называется банаховым пространством.

Пусть в пространстве $X$ дано некоторое точечное множество $D$. Напомним некоторые определения из [2]. Всякая функция $T$, которая каждому элементу $f \in D$ относит некоторый элемент $T f=g \in \Delta \subseteq X$, называется оператором в пространстве $X$ с областью определения $D$ и множеством значений $\Delta$, состоящим из всех $g=T f$, где $f$ пробегает всё $D$.

Оператор $T$ называется линейным, если его область определения $D$ есть линейное многообразие и

$$
T(\alpha f+\beta g)=\alpha T f+\beta T g
$$

для любых $f, g \in D$ и любых комплексных $\alpha, \beta$.

Линейный оператор $T$ назовем ограниченным, если

$$
\sup \{\|T f\|: f \in D,\|f\| \leqslant 1\}<\infty .
$$

Норма ограниченного линейного оператора $T$ определяется равенством

$$
\|T\|=\sup \{\|T f\|: f \in D,\|f\|=1\} .
$$

Обозначим $L(X)$ множество ограниченных линейных операторов, определенных всюду в банахово пространстве $X$. Если $S, T \in L(X)$, то операторы $S T$ и $T S$ также являются ограниченными линейными операторами, определенными всюду в $X$, при этом

$$
\|S T\| \leqslant\|S\|\|T\|, \quad\|T S\| \leqslant\|T\|\|S\| .
$$


Пусть $\tilde{A}=A_{1}, A_{2}, \ldots$ - последовательность операторов из $L(X)$. Будем называть

$$
F(\tilde{A})=\prod_{k=1}^{n} A_{k}
$$

частичным произведением последовательности ограниченных линейных операторов.

\section{2. Основные результаты}

Теорема 1. Пусть $\tilde{A}=A_{1}, A_{2}, \ldots u \tilde{A}^{\prime}=A_{1}^{\prime}, A_{2}^{\prime}, \ldots$ - две последовательности операторов из $L(X)$. Пусть члень последовательности обладают следующими свойствами:

(а) $A_{i} A_{i+1}^{\prime}=A_{i}^{\prime} A_{i+1}=A_{i}^{\prime} A_{i+1}^{\prime}$ при любом $i \in \mathbf{N}$;

(б) $\left\|A_{i}-A_{i}^{\prime}\right\| \leqslant \lambda$ при любом $i \in \mathbf{N}$.

Тогда

$$
\left\|F_{n}(\tilde{A})-F_{n}\left(\tilde{A}^{\prime}\right)\right\| \leqslant \lambda^{n}
$$

Доказательство. Раскрыв скобки в выражении $\prod_{k=1}^{n}\left(A_{k}-A_{k^{\prime}}\right)$ и воспользовавшись условием (а) теоремы, получим, что

$$
\prod_{k=1}^{n}\left(A_{k}-A_{k}^{\prime}\right)=\prod_{k=1}^{n} A_{k}-\prod_{k=1}^{n} A_{k}^{\prime} .
$$

Отсюда следует, что

$$
\left.\| F_{n}(\tilde{A})-F\right) n\left(\tilde{A^{\prime}}\right)\left\|\leqslant \prod_{k=1}^{n}\right\| A_{k}-A_{k}^{\prime} \| .
$$

Используя условие (б), получаем оценку

$$
\left.\| F_{n}(\tilde{A})-F\right) n\left(\tilde{A}^{\prime}\right) \| \leqslant \lambda^{n}
$$

что и требовалось доказать.

Гильбертово пространство - векторное пространство $H$ над полем комплексных (или действительных) чисел вместе с комплексной (действительной) функцией $(x, y)$, определенной на $H \times H$ и обладающей следующими свойствами:

(1) $(x, y)=0$ в том и только в том случае, если $x=0$;

(2) $(x, x) \geqslant 0$ для всех $x \in H$;

(3) $(x+y, z)=(x, z)+(y, z), x, y, z \in H$;

(4) $(\alpha x, y)=\alpha(x, y), x, y \in H, \alpha$ - комплексное число;

(5) $(x, y)=\overline{(y, x)}, x, y \in H$; 
(6) если $x_{n} \in H, n=1,2, \ldots$, и

$$
\lim _{n, m \rightarrow \infty}\left(x_{n}-x_{m}, x_{n}-x_{m}\right)=0,
$$

то существует такой элемент $x \in H$, что

$$
\lim _{n \rightarrow \infty}\left(x-x_{n}, x-x_{n}\right)=0
$$

элемент $x$ называется пределом последовательности $x_{n} \in H$;

(7) $H$ - бесконечное векторное пространство.

Пространство называется сепарабельным, если оно содержит счетное всюду плотное множество.

Всюду ниже мы будем рассматривать сепарабельные гильбертовы пространства. Сингулярными числами оператора $K$ называются неотрицательные квадратные корни собственных чисел оператора $K^{*} K$, где $K^{*}-$ оператор, сопряженный к $K$. Сингулярные числа мы всегда будем располагать в порядке возрастания с учетом кратности (см. [1]).

Сингулярное число оператора $K$ с номером $i$ будем обозначать $\sigma_{i}(K)$.

Определенный всюду в $H$ линейный оператор $A$ линейный оператор $A$ называется вполне непрерывным, если он переводит всякое ограниченное множество точек в множество, компактное в смысле сильной сходимости (см. [1]). Множество определенных всюду в $H$ вполне непрерывных операторов будем обозначать $H_{\infty}$.

Оценка сингулярных чисел вполне непрерывных операторов, полученных при помощи произведения или суммы других операторов изучалась во многих работах. Обзоры результатов можно найти в $[2,3]$. Тем не менее, задача оценки сингулярных чисел линейных операторов, полученных при помощи других действий, остается по-прежнему актуальной.

Для дальнейшего изложения нам потребуется следующая лемма.

Лемма 1. Пусть $K=K_{1} K_{2} \cdots K_{m}$, где $K_{i} \in H_{\infty}$ для всех $i \in\{1, \ldots, m\}, \sigma_{j}\left(K_{i}\right) \leqslant \alpha_{i}$ для всех $j \in \mathbf{N}, i=\{1, \ldots, m\}$.

Пусть также для некоторого $и>0$ ряд $\sum_{i} \alpha_{i}^{u}$ сходится.

Пусть $\gamma_{1}, \gamma_{2}, \ldots$ - сингулярные числа оператора $K$.

Тогда ряд $\sum_{i} \gamma_{i}^{u}$ сходится $и$

$$
\sum_{i} \gamma_{i}^{u} \leqslant \sum_{i} \alpha_{i}^{u m} .
$$

Доказательство. Из теоремы Хорна [2] следует что для сингулярных чисел $\gamma_{1}, \gamma_{2}, \ldots$ произведения вполне непрерывных операторов $A$ и $B$ с сингулярными числами $\alpha_{1}, \alpha_{2}, \ldots$ и $\beta_{1}, \beta_{2}, \ldots$, соответственно, для любого $u>0$ и любого $n \in \mathbf{N}$ верно неравенство

$$
\sum_{i=1}^{n} \gamma_{i}^{u} \leqslant \sum_{i=1}^{n} \alpha_{i}^{u} \beta_{i}^{u}
$$

Устремив $n$ к бесконечности, получим, что

$$
\sum_{i} \gamma_{i}^{u} \leqslant \sum_{i} \alpha_{i}^{u} \beta_{i}^{u} .
$$


Докажем утверждение леммы индукцией по $m$. При $m=1$ утверждение леммы очевидно. Пусть оно выполнено для $m-1, m \geqslant 2$, докажем его для $m$. Обозначим $\beta_{1}, \beta_{2}, \ldots$ сингулярные числа $K_{2} \cdots K_{m}$. Из соотношения, доказанного выше, следует, что

$$
\sum_{i} \gamma_{i}^{u} \leqslant \sum_{i} \alpha_{i}^{u} \beta_{i}^{u}
$$

Рассмотрим ряды $\sum_{i} \alpha_{I}^{q u}$ и $\sum_{i} \beta_{i}^{p u}$, где $p=m /(m-1), q=$. Так как $p>1$ и $q>1$, ряды сходятся и поскольку $p^{-1}+q^{-1}=1$, к правой части вышеприведенного соотношения можно применить неравенство Гельдера, откуда следует, что

$$
\sum_{i} \gamma_{i}^{u} \leqslant\left(\sum_{i} \beta_{i}^{p u}\right)^{1 / p}\left(\sum_{i} \alpha_{i}^{q u}\right)^{1 / q}
$$

По предположению индукции, для первого сомножителя в правой части справедлива оценка

$$
\left(\sum_{i} \beta_{i}^{p u}\right)^{1 / p} \leqslant\left(\sum_{i} \alpha_{i}^{p u(m-1)}\right)^{(m-1) / m}=\left(\sum_{i} \alpha_{i}^{m u}\right)^{1-1 / m} .
$$

Для второго сомножителя в правой части справедливо равенство

$$
\left(\sum_{i} \alpha_{i}^{q u}\right)^{1 / q}=\left(\sum_{i} \alpha^{m u}\right)^{1 / m} \text {. }
$$

Перемножив, получаем, что

$$
\sum_{i} \gamma_{i}^{u} \leqslant \sum_{i} \alpha_{i}^{u m}
$$

что и требовалось доказать.

Для конечномерных матриц утверждение леммы доказано в [6].

Теорема 2. Пусть $\tilde{A}=A_{1}, A_{2}, \ldots u \tilde{A}^{\prime}=A_{1}^{\prime}, A_{2}^{\prime}, \ldots$ - последовательности операторов из $L(H)$, и пусть члены последовательностей обладают следующими свойствами:

(а) $A_{i} A_{i+1}^{\prime}=A_{i}^{\prime} A_{i+1}=A_{i}^{\prime} A_{i+1}^{\prime}$ при любом $i \in \mathbf{N}$;

(б) $\left(A_{j}-A_{j}^{\prime}\right) \in H_{\infty} u \sigma_{i}\left(A_{j}-A_{j}^{\prime}\right) \leqslant \alpha_{i}$ при любых $i, j \in \mathbf{N}$;

(в) для некоторого $и>0$ ряд $\sum_{i} \alpha_{i}^{u}$ сходится.

Пусть $\gamma_{1}, \gamma_{2}, \ldots$ - сингулярные числа оператора $F_{n}(\tilde{A})-F_{n}\left(\tilde{A}^{\prime}\right)$.

Тогда ряд $\sum_{i} \gamma_{i}^{u}$ сходится, $u$

$$
\sum_{i} \gamma_{i}^{u} \leqslant \sum_{i} \alpha_{i}^{n u}
$$

Пусть $A$ - ограниченный линейный оператор, определенный всюду в сепарабельном гильбертовом пространстве $H$, и пусть $\left\{f_{k}\right\}_{1}^{\infty},\left\{e_{k}\right\}_{1}^{\infty}-$ ортонормированные базисы в $H$. Если ряд $\sum_{i, k}\left|\left(A f_{k}, e_{i}\right)\right|^{2}$ сходится, то величину

$$
N(A)=\sqrt{\sum_{i, k}\left|\left(A f_{k}, e_{i}\right)\right|^{2}}
$$

назовем абсолютной нормой оператора $A$. 
Следствие 1. Пусть $\tilde{A}=A_{1}, A_{2}, \ldots u \tilde{A}^{\prime}=A_{1}^{\prime}, A_{2}^{\prime}, \ldots$ - последовательности операторов из $L(H)$, и пусть члены последовательностей обладают следующими свойствами:

(а) $A_{i} A_{i+1}^{\prime}=A_{i}^{\prime} A_{i+1}=A_{i}^{\prime} A_{i+1}^{\prime}$ при любом $i \in \mathbf{N}$;

(б) $\left(A_{j}-A_{j}^{\prime}\right) \in H_{\infty}$ и $\sigma_{i}\left(A_{j}-A_{j}^{\prime}\right) \leqslant \lambda_{i}$ при любых $i, j \in \mathbf{N}$;

(в) ряд $\sum_{i} \alpha_{i}^{2}$ сходится.

Тогда

$$
N\left(F_{n}(\tilde{A})-F_{n}\left(\tilde{A}^{\prime}\right)\right) \leqslant \sqrt{\sum_{j} \lambda_{j}^{2 n}} .
$$

Пользуясь методом, использованным в [2] для доказательства теоремы 4, получаем следующую теорему.

Теорема 3. Пусть $\tilde{A}=A_{1}, A_{2}, \ldots$ и $\tilde{A}_{1}^{\prime}=A_{1}^{\prime}, A_{2}^{\prime}, \ldots$-последовательности операторов из $L(H)$, и пусть члены последовательностей обладают следующими свойствами:

(а) $A_{i} A_{i+1}^{\prime}=A_{i}^{\prime} A_{i+1}=A_{i}^{\prime} A_{i+1}^{\prime}$ при любом $i \in \mathbf{N}$;

(б) $\left(A_{j}-A_{j}^{\prime}\right) \in H_{\infty} u \sigma_{i}\left(A_{j}-A_{j}^{\prime}\right) \leqslant \alpha_{i}$ при любых $i, j \in \mathbf{N}$;

(в) для некоторого $и>0$ ряд $\sum_{i} \alpha_{i}^{u}$ сходится.

Пусть $\gamma_{1}, \gamma_{2}, \ldots$ - сингулярные числа оператора $F_{n}(\tilde{A})-F_{n}\left(\tilde{A}^{\prime}\right)$.

Тогда ряд $\sum_{i} \gamma_{i}^{u / n}$ сходится. $и$

$$
\sum_{i} \gamma_{i}^{u / n} \leqslant \sum_{i} \alpha_{i}^{u}
$$

\section{3. Пример применения}

Рассмотрим следующее применение полученных результатов. Рассмотрим пространство $L^{2}(a, b)$ (см. [3], с. 174). Элементами $L^{2}(a, b)$ являются классы эквивалентных между собой (совпадающих почти всюду) действительных, квадратично-суммируемых функций, определенных на $(a, b)$. Норма элемента $f(x)$ пространства $L_{2}(a, b)$ есть

$$
\|f\|=\sqrt{\int_{a}^{b} f^{2}(x) d x} .
$$

Пусть на $(a, b) \times(a, b)$ задана квадратично-суммируемая функция $K(x, s), a \leqslant x, s \leqslant b$. Отображение $A: L^{2}(a, b) \rightarrow L^{2}(a, b)$, определяемое формулой

$$
A f(x)=\int_{a}^{b} K(x, s) f(s) d s
$$

является ограниченным линейным оператором. Интегральные уравнения Фредгольма второго рода

$$
\varphi(x)-\lambda \int_{a}^{b} K(x, s) \varphi(s) d s=f(x)
$$


при условиях, что ядро $K(x, s), a \leqslant x, s \leqslant b$, уравнения квадратично-суммируемо и $|\lambda|<B^{-1}$, где

$$
B^{2}=\int_{a}^{b} \int_{a}^{b} K^{2}(x, s) d x d s
$$

$f(x) \in L^{2}(a, b)$, имеют единственное квадратично-суммируемое решение. Для нахождения решения используется метод последовательных приближений

$$
\begin{aligned}
\varphi_{0}(x) & =f(x), \\
\varphi_{1}(x) & =f(x)+\lambda \int_{a}^{b} K(x, s) \varphi_{0}(s) d s, \\
& \ldots \\
\varphi_{n+1}(x) & =f(x)+\lambda \int_{a}^{b} K(x, s) \varphi_{n}(s) d s,
\end{aligned}
$$

при $|\lambda| \leqslant B^{-1}$ последовательность $\left\{\varphi_{n}(x)\right\}$ равномерно сходится к решению уравнения.

Этот метод представим посредством итерированных ядер $K_{n}(x, s)$ :

$$
\begin{aligned}
K_{1}(x, s) & =K(x, s), \\
K_{n+1}(x, s) & =\int_{a}^{b} K_{n}(x, z) K(z, s) d z .
\end{aligned}
$$

Очевидно, что отображение функции $f(x) \in L^{2}(a, b)$, заданное посредством формулы $\int_{a}^{b} K_{n}(x, s) f(s) d s$, есть $A^{n} f(x)$.

Зачастую ядро уравнения известно с некоторой погрешностью. Рассмотрим второе уравнение

$$
\varphi^{\prime}(x)-\lambda \int_{a}^{b} K^{\prime}(x, s) \varphi^{\prime}(s) d s=f(x) .
$$

Ядро $K^{\prime}(x, s), a \leqslant x, s \leqslant b$, удовлетворяет следующим условиям: оно квадратичносуммируемо и

$$
\begin{gathered}
\int_{a}^{b} \int_{a}^{b}\left(K^{\prime}(x, s)\right)^{2} d x d s=B^{2} \\
\int_{a}^{b} K(x, z) K^{\prime}(z, x) d z=\int_{a}^{b} K^{\prime}(x, z) K(z, s) d z=\int_{a}^{b} K^{\prime}(x, z) K^{\prime}(z, s) d z .
\end{gathered}
$$

Зададим оператор $A^{\prime}: L^{2}(a, b) \rightarrow L^{2}(a, b)$ формулой

$$
A^{\prime} f(x)=\int_{a}^{b} K^{\prime}(x, s) f(s) d s .
$$

Пусть также выполняется условие

$$
\left\|A-A^{\prime}\right\| \leqslant \mu .
$$

Тогда последовательности линейных ограниченных операторов $\tilde{A}=A, A, \ldots$ и $\tilde{A}^{\prime}=A^{\prime}, A^{\prime}, \ldots$ удовлетворяют условиям теоремы 1 , и норму разности между решением 
одного уравнения и другого можно оценить следующим образом:

$$
\begin{aligned}
\left\|\varphi(x)-\varphi^{\prime}(x)\right\| & =\left\|\int_{a}^{b}\left(\sum_{n=1}^{\infty} \lambda^{n} K_{n}(x, s)\right) f(s) d s-\int_{a}^{b}\left(\sum_{n=1}^{\infty} \lambda^{n} K_{n}^{\prime}(x, s)\right) f(s) d s\right\| \\
& =\left\|\sum_{n=1}^{\infty} \lambda^{n} F_{n}(\tilde{A}) f(x)-\sum_{n=1}^{\infty} \lambda^{n} F_{n}\left(\tilde{A}^{\prime}\right) f(x)\right\| \\
& \leqslant \sum_{n=1}^{\infty}|\lambda|^{n}\left\|F_{n}(\tilde{A})-F_{n}\left(\tilde{A}^{\prime}\right)\right\|\|f\| \\
& \leqslant \sum_{n=1}^{\infty}|\lambda|^{n} \mu^{n}\|f\|=\|f\| \frac{1}{1-|\lambda| \mu}
\end{aligned}
$$

при $|\lambda| \mu<1$.

\section{Список литературы}

1. Ахиезер Н. И., Глазман И. М., Теория линейных операторов в гильбертовом пространстве. Наука, Москва, 1966.

2. Horn A., On the singular values of a product of completely continuous operators. Proc. Natl. Acad. USA (1950) 36, №7, 374-375.

3. Садовничий В. А., Теория операторов. Дрофа, Москва, 2001.

4. Маркус А. С., Собственные и сингулярные числа суммы и произведения линейных операторов. Успехи матем. наук (1964) 19, №4, 93-123.

5. Ефимов Д. Е., Оценка экспонента некоторых множеств неотрицательных матриц. Дискретная математика (1990) 11, №4, 79-88.

Статья поступила 21.10.2011. 